\title{
Suplementación de $\beta$-caroteno en dietas balanceadas con exclusión de forraje en cuyes (Cavia porcellus) reproductoras hembras
}

\author{
ß-CAROTENE SUPPLEMENTATION IN NON-FORAGE DIETS IN FEMALE BREEDING GUINEA PIGS \\ (Cavia porcellus)
}

\author{
Carla Bustios M. ${ }^{1}$, Víctor Vergara R. ${ }^{1,2}$, Lili Chauca F. ${ }^{1}$
}

\section{Resumen}

El objetivo del estudio fue evaluar la suplementación de $\beta$-caroteno en dietas con exclusión de forraje verde de cuyes hembras en etapa reproductiva. El experimento I se realizó en primavera (octubre-diciembre) con 27 hembras primerizas y el experimento II se hizo en el verano (enero-abril), con las mismas hembras durante su segundo parto. Se evaluaron tres dietas: mixta (forraje verde + concentrado), integral (solo concentrado) e integral $+\beta$-caroteno. La suplementación de $\beta$-caroteno no mejoró los parámetros productivos ni reproductivos en las hembras en ambos experimentos. Los resultados del segundo parto fueron afectados por el verano, donde se pudo observar el rol benéfico del $\beta$-caroteno como antioxidante, natural o sintético, en el peso de las madres al destete.

Palabras clave: $\beta$-caroteno; cuyes hembras; reproducción; primavera; verano

\section{AbSTRACT}

The objective of the study was to evaluate the $\beta$-carotene supplementation in nonforage diets of female breeding guinea pigs. Experiment I was carried out in the spring (October-December) with 27 females at the first parturition and experiment II was carried out in the summer (January-April), with the same females during their second parturition. Three diets were evaluated: mixed (green forage + concentrated), integral (only concentrated) and integral $+\beta$-carotene. The supplementation of $\beta$-carotene did not

\footnotetext{
${ }^{1}$ Programa de Investigación y Proyección Social en Alimentos, Facultad de Zootecnia, Universidad Nacional Agraria La Molina, Lima, Perú

${ }^{2}$ E-mail: vjvergara@lamolina.edu.pe
}

Recibido: 15 de octubre de 2017

Aceptado para publicación: 16 de junio de 2018 
improve the productive or reproductive parameters in guinea pigs in both experiments. The results of the second parturition were affected by the summer, showing the beneficial role of $\beta$-carotene as an antioxidant, natural or synthetic, in body weight of mothers at weaning.

Key words: $\beta$-carotene; female; guinea pigs; reproduction; spring; summer

\section{INTRODUCCIÓN}

La disponibilidad de pastos y forrajes para la crianza de cuyes se encuentra afectada por las frecuentes sequías en la sierra peruana y por la restricción del cultivo y el extremo calor en la costa. Es así que surgió como alternativa la alimentación basada en concentrados (alimentación integral), y que permite cubrir los requerimientos nutritivos del animal (Solórzano, 2014a). Este sistema de alimentación tiene un efecto positivo en cuyes en crecimiento, pero en la etapa reproductiva no se le considera como alternativa viable, dada la frecuencia de abortos y nacidos muertos que produce la exclusión de forraje (Mamani, 2016; Pedraz, 2001; Revilla, 2011).

Se estima que un posible nutriente que podría estar faltando en el concentrado, pero que está presente en el forraje verde, es el $\beta$-caroteno (precursor de la vitamina A) (Pickworth et al., 2011), que cumple un rol importante en la reproducción. Su suplementación ha demostrado efectos favorables en el fluido folicular en conejos, ratas, marranas y vacas (Chew et al., 1984; Haliloglu et al., 1984; Schweigert y Zucker, 1988), donde una parte se transforma en retinol, considerado como una fuente extra de vitamina A, y la otra parte está asociada a su acción antioxidante de estímulo de los niveles de progesterona, cuyos efectos no pueden ser reemplazados con la suplementación de vitamina A (Graves-Hoagland, 1998).
Se ha comprobado que el estrés por calor reduce de forma considerable la concepción (Badinga et al., 1985) y aumenta la mortalidad embrionaria (Putney et al., 1989). Esto es debido al incremento de la producción de radicales libres que pueden retardar o bloquear el desarrollo del embrión (Loven, 1988), o por una reducción de la actividad de los sistemas antioxidantes del organismo (Aréchiga et al., 1995). La suplementación continua con $\beta$-caroteno suprime los efectos negativos del estrés por calor (Aréchiga et al., 1998).

El $\beta$-caroteno tiene una estrecha relación con la tasa de concepción y la mortalidad embrionaria. Como estimulante de los niveles de progesterona, los niveles de $\beta$ caroteno y progesterona están directamente asociados a la permanencia del cuerpo lúteo. Niveles bajos del primero disminuyen la concentración de progesterona en las primeras etapas del desarrollo embrionario, retrasando el crecimiento del embrión, afectando el reconocimiento materno de la gestación, por lo que ocurre la lisis del cuerpo lúteo y la muerte del embrión (Wathes et al., 2003).

El presente trabajo tuvo como objetivo evaluar la suplementación de $\beta$-caroteno en un sistema de alimentación integral con exclusión de forraje verde en cuyes reproductoras en la etapa de gestación y lactación, durante el primer y segundo parto, midiendo las características productivas y reproductivas. 


\section{Materiales y Métodos}

La investigación se realizó en las instalaciones del Programa Nacional de Investigación de Cuyes del Instituto Nacional de Innovación Agraria (INIA), La Molina, Lima. La preparación de las dietas experimentales se realizó en la Planta de Alimentos Balanceados del Programa de Investigación y Proyección Social en Alimentos, Facultad de Zootecnia, de la Universidad Nacional Agraria La Molina en Lima, Perú.

Se realizó un primer experimento entre agosto y diciembre de 2015, con una etapa de crecimiento y adaptación de los cuyes hasta octubre y el periodo que abarcó la gestación y lactación entre octubre y diciembre, correspondiendo a la época de primavera. El segundo experimento se realizó entre enero y abril de 2016 (meses de verano), que correspondió a la gestación y lactación del segundo parto de los cuyes empleados en el experimento I. El rango de las temperaturas entre octubre y diciembre fue de $18-25^{\circ} \mathrm{C}$ y entre enero y abril fue de $23-31{ }^{\circ} \mathrm{C}$. La temperatura se midió con un termómetro digital ubicado al centro del galpón, a $10 \mathrm{~cm}$ sobre la cama, registrándose los valores máximos y mínimos durante el día.

\section{Animales}

Para determinar el tamaño de muestra se usó la fórmula para poblaciones finitas. En el Experimento I se utilizaron 27 cuyes hembras (30 \pm 2 días y peso aproximado de $500 \mathrm{~g}$ ) de línea comercial obtenida del cruce de las razas Perú, Inti y Andina y 9 cuyes machos del plantel de reproductores para empadrar a las hembras primerizas. En el Experimento II se utilizaron las mismas hembras, pero en su segundo parto. Los cuyes fueron distribuidos en nueve pozas (cada una considerada una unidad experimental), siendo tres pozas por tratamiento, a razón de tres hembras y un macho por poza.

\section{Tratamientos}

Se evaluaron tres dietas con tres repeticiones por tratamiento en ambos experimentos). T1: dieta integral $\sin \beta$-caroteno + forraje verde; $\mathrm{T} 2$ : dieta integral $\sin \beta$-caroteno y sin forraje verde; $T 3$ : dieta integral $+30 \mathrm{mg}$ $\beta$-caroteno $/ \mathrm{kg}$ y $\sin$ forraje verde. El nivel de $\beta$-caroteno utilizado fue determinado teniendo como referencia recomendaciones establecidas para dietas en cuyes (Lab. Diet, 2014) $\mathrm{y}$ conejos (Besenfelder et al., 1995).

En el Experimento I se diseñaron seis dietas experimentales, de las cuales tres fueron para la adaptación al nuevo alimento con dietas de reemplazo de acuerdo con el estudio realizado por Enciso (2010), y las otras tres dietas para la parte experimental (reproducción), utilizando la formulación al mínimo costo. Las dietas fueron peletizadas en un diámetro de $4.5 \mathrm{~mm}$ y $12 \mathrm{~mm}$ de longitud. Para el Experimento II, se utilizaron las mismas dietas de reproductoras del Experimento I. En ambos casos, para asegurar la inclusión uniforme del $\beta$-caroteno, se realizó una premezclado durante 10 minutos del $\beta$ caroteno con los demás aditivos, utilizando el subproducto de trigo como vehículo. Luego de obtenida la premezcla se procedió a mezclarla con los demás ingredientes y aditivos que conformaban el alimento.

\section{Alimentación}

En ambos experimentos, el forraje verde utilizado fue chala, proveniente del INIA, cortado en las tardes, dejándose orear por aproximadamente 12 horas. Fue proporcionado en las mañanas y en cantidades equivalentes al 10\% del peso vivo. En el Experimento 1 fue de aproximadamente $85 \mathrm{~g} /$ cuy/día en el empadre, $150 \mathrm{~g} /$ cuy/día durante la gestación, $130 \mathrm{~g} /$ cuy/día durante la lactación, y en el Experimento II se proporcionó $130 \mathrm{~g} /$ cuy/día durante el empadre, $200 \mathrm{~g} /$ cuy/día durante la gestación y de $150 \mathrm{~g} /$ cuy/día durante la lactación. El residuo fue retirado al final 
del día. El suministro de agua se realizó en las mañanas (08:00) y en las tardes (15:00), y en los meses de verano se hizo, además, al mediodía (12:00). Los bebederos fueron lavados y desinfectados diariamente para evitar problemas sanitarios.

\section{Parámetros Evaluados}

Se evaluó el porcentaje de fertilidad y de abortos, el tamaño de camada al nacimiento $y$ al destete, pesos al nacimiento y destete en la progenie, mortalidad de crías al nacimiento y al destete, así como el peso vivo al empadre, parto y destete y mortalidad de las madres. Los controles de pesos de los animales y del alimento balanceado se realizaron en una balanza digital de $5 \mathrm{~kg}$ de capacidad con precisión de $2 \mathrm{~g}$.

\section{Análisis Estadístico}

Se utilizó el análisis de variancia para determinar las diferencias significativas entre tratamientos (á $=0.05$ ), bajo un diseño completamente al azar con tres tratamientos y tres repeticiones por tratamiento. Se utilizó la prueba de Tukey para determinar las diferencias entre medias de los tratamientos.

\section{Resultados y Discusión}

El consumo de materia seca por día por reproductora presentó diferencias significativas $(p<0.05)$ entre tratamientos en ambos experimentos. Los cuyes de T1 (integral + forraje) presentaron el mayor consumo de materia seca (95.58 g/día), posiblemente debido a que los cuyes complementan su alimentación con el aporte del forraje verde.

Asimismo, se encontró una depresión significativa en el consumo del alimento balanceado en el verano (Experimento II), siendo inferior en 37,33 y $31 \%$ con relación a la primavera (Experimento I) para los tres tratamientos, respectivamente, debido posiblemente al estrés por calor en el verano. Chauca et al. (2012) indican que el consumo de alimento de los cuyes en verano fue de

Cuadro 1. Efecto de la suplementación de $\beta$-caroteno en la dieta sobre la eficiencia reproductiva de cuyes de primer parto (promedio \pm desviación estándar)

\begin{tabular}{|c|c|c|c|c|}
\hline \multirow{2}{*}{ Parámetros reproductivos } & \multicolumn{3}{|c|}{ Tratamientos } & \multirow{2}{*}{ P-value } \\
\hline & 1 & 2 & 3 & \\
\hline Fertilidad (\%) & $100^{\mathrm{a}}$ & $100^{\mathrm{a}}$ & $100^{\mathrm{a}}$ & \\
\hline Abortos (\%) & 0 & 0 & 0 & \\
\hline $\begin{array}{l}\text { Tamaño de camada total } \\
\text { al nacimiento }\end{array}$ & $4.27 \pm 1.02^{\mathrm{a}}$ & $3.31 \pm 0.44^{\mathrm{a}}$ & $3.21 \pm 1.29^{\mathrm{a}}$ & 0.40 \\
\hline $\begin{array}{l}\text { Tamaño de camada de } \\
\text { crías vivas al nacimiento }\end{array}$ & $4.01 \pm 0.84^{\mathrm{a}}$ & $3.13 \pm 0.95^{\mathrm{a}}$ & $3.00 \pm 1.61^{\mathrm{a}}$ & 0.45 \\
\hline $\begin{array}{l}\text { Tamaño de camada al } \\
\text { destete }\end{array}$ & $3.50 \pm 0.38^{\mathrm{a}}$ & $3.13 \pm 0.95^{\mathrm{a}}$ & $3.00 \pm 1.62^{\mathrm{a}}$ & 0.78 \\
\hline $\begin{array}{l}\text { Mortalidad de crías al } \\
\text { nacimiento }(\%)\end{array}$ & $4.79 \pm 5.59^{\mathrm{a}}$ & $15.83 \pm 17.35^{\mathrm{a}}$ & $15.28 \pm 23.12^{\mathrm{a}}$ & 0.62 \\
\hline $\begin{array}{l}\text { Mortalidad de crías al } \\
\text { destete (\%) }\end{array}$ & $12.7 \pm 15.0^{\mathrm{a}}$ & $0^{\text {a }}$ & $0^{\text {a }}$ & 0.09 \\
\hline
\end{tabular}

a Letras similares dentro de filas indican ausencia de diferencia estadística 
Cuadro 2. Efecto de la suplementación de $\beta$-caroteno en la dieta sobre el comportamiento productivo de cuyes de primer parto

\begin{tabular}{|c|c|c|c|c|}
\hline \multirow{2}{*}{ Parámetros productivos } & \multicolumn{3}{|c|}{ Tratamientos } & \multirow{2}{*}{ P-value } \\
\hline & $\mathrm{T} 1$ & $\mathrm{~T} 2$ & $\mathrm{~T} 3$ & \\
\hline \multicolumn{5}{|l|}{ Madres } \\
\hline $\begin{array}{l}\text { - Número de hembras } \\
\text { empadradas }\end{array}$ & 9 & 9 & 9 & \\
\hline $\begin{array}{l}\text { - Número de hembras que } \\
\text { parieron }\end{array}$ & 8 & 8 & 8 & \\
\hline - Peso al empadre (g) & $823 \pm 77.64^{\mathrm{a}}$ & $830 \pm 44.80^{\mathrm{a}}$ & $816 \pm 64.06^{\mathrm{a}}$ & 0.97 \\
\hline - Peso al parto (g) & $1327 \pm 114.82^{\mathrm{a}}$ & $1237 \pm 197.38^{a}$ & $1270 \pm 40.33^{\mathrm{a}}$ & 0.72 \\
\hline - Peso al destete (g) & $1352 \pm 85.44^{\mathrm{a}}$ & $1230 \pm 214.37$ & $1288 \pm 62.26^{\mathrm{a}}$ & 0.59 \\
\hline - Mortalidad (\%) & $11.11 \pm 0.19^{\mathrm{a}}$ & $22.22 \pm 0.19^{\mathrm{a}}$ & $11.11 \pm 0.19^{\mathrm{a}}$ & 0.73 \\
\hline \multicolumn{5}{|l|}{ Crías } \\
\hline - Peso al nacimiento (g) & $142 \pm 12^{\mathrm{a}}$ & $138 \pm 15^{\mathrm{a}}$ & $165 \pm 37^{\mathrm{a}}$ & 0.39 \\
\hline - Peso al destete (g) & $283 \pm 39^{\mathrm{a}}$ & $259 \pm 17^{\mathrm{a}}$ & $292 \pm 50^{\mathrm{a}}$ & 0.58 \\
\hline
\end{tabular}

a Letras similares dentro de filas indican ausencia de diferencia estadística

$70.25 \mathrm{~g} /$ día, mientras que en el invierno fue de $85.54 \mathrm{~g} /$ día. Por otro lado, Vergara (2008) reporta consumos promedio de $85 \mathrm{~g} /$ día en condiciones normales de temperatura.

\section{Experimento I}

No se encontraron diferencias significativas entre tratamientos en los parámetros reproductivos (Cuadro 1). No obstante, se observó una aparente mejor respuesta biológica en T1 (cuyes alimentados con forraje verde más concentrado), resultados que concuerdan con el estudio de Alejandro (2016). La mortalidad al destete se produjo solo en $\mathrm{T} 1$, posiblemente debido a la exposición de las crías al forraje verde, toda vez que el sistema inmunológico de las crías no está totalmente desarrollado, presentándose diarreas y muerte. Al respecto Chauca (2016) indica que la mayor mortalidad de crías se da entre los días 1 y 4 de nacidos.

No se encontraron diferencias significativas entre tratamientos en los parámetros productivos (Cuadro 2). Tal y como se ob- servó en el caso de la eficiencia reproductiva, los cuyes de T1 presentaron una aparente mejor respuesta biológica. Por otro lado, los cuyes del T3 ( $\beta$-caroteno) mostraron asimismo una aparente mejor respuesta en varias de las variables en comparación con T2 ( sin $\beta$-caroteno natural o sintético), especialmente en el peso del parto y al destete. Similares resultados fueron reportados por Mamani (2016) en cuyes primerizas. Estos resultados probablemente estén relacionados con el efecto antioxidante del $\beta$-caroteno presente en el forraje verde, lo cual favorece el metabolismo en los animales.

\section{Experimento II}

No se encontraron diferencias significativas entre tratamientos en los parámetros reproductivos (Cuadro 3 ) en este experimento, donde la gestación y la lactación ocurrió durante los meses de verano. No obstante, se observó una aparente mejor respuesta biológica en T1 y T3 (cuyes alimentados con forraje verde o suplemento de $\beta$-caroteno más concentrado). Los valores obtenidos fueron 
Cuadro 3. Efecto de la suplementación de $\beta$-caroteno en la dieta sobre la eficiencia reproductiva de cuyes de segundo parto

\begin{tabular}{lcccc}
\hline Parámetros reproductivos & \multicolumn{3}{c}{ Tratamientos } & $\begin{array}{c}\text { P- } \\
\text { value }\end{array}$ \\
\cline { 2 - 4 } & $100^{\mathrm{a}}$ & 2 & 3 & $100^{\mathrm{a}}$ \\
\hline Fertilidad (\%) & $16.7 \pm 28.9^{\mathrm{a}}$ & $16.7 \pm 28.9^{\mathrm{a}}$ & $0^{\mathrm{a}}$ & 0.63 \\
$\begin{array}{l}\text { Abortos (\%) } \\
\text { Tamaño de camada total al }\end{array}$ & $3.78 \pm 0.51^{\mathrm{a}}$ & $3.11 \pm 1.00^{\mathrm{a}}$ & $3.06 \pm 0.42^{\mathrm{a}}$ & 0.48 \\
$\begin{array}{l}\text { nacimiento } \\
\text { Tamaño de camada de crías }\end{array}$ & $2.90 \pm 0.19^{\mathrm{a}}$ & $2.43 \pm 0.50^{\mathrm{a}}$ & $2.50 \pm 0.10^{\mathrm{a}}$ & 0.22 \\
$\begin{array}{l}\text { vivas al nacimiento } \\
\begin{array}{l}\text { Tamaño de camada al } \\
\text { destete }\end{array}\end{array}$ & $2.22 \pm 0.69^{\mathrm{a}}$ & $1.60 \pm 0.67^{\mathrm{a}}$ & $1.89 \pm 0.19^{\mathrm{a}}$ & 0.45 \\
$\begin{array}{l}\text { Mortalidad de crías al } \\
\text { nacimiento (\%) }\end{array}$ & $22.8 \pm 12.9^{\mathrm{a}}$ & $14.4 \pm 10.1^{\mathrm{a}}$ & $19.3 \pm 6.1^{\mathrm{a}}$ & 0.72 \\
$\begin{array}{l}\text { Mortalidad de crías al } \\
\text { destete (\%) }\end{array}$ & $23.6 \pm 26.7^{\mathrm{a}}$ & $37.4 \pm 12.7^{\mathrm{a}}$ & $22.9 \pm 6.4^{\mathrm{a}}$ & 0.44 \\
\hline
\end{tabular}

a Letras similares dentro de filas indican ausencia de diferencia estadística

inferiores a los registrados durante el primer parto, probablemente debido a la mayor temperatura ambiental del verano.

Los cuadros de abortos se presentaron en T1 y T2 (equivalente a una hembra por tratamiento). Besenfelder et al. (1995) resaltan el efecto del $\beta$-caroteno en el mantenimiento de la gestación a través de la permanencia del cuerpo lúteo; no obstante, en este estudio, los cuyes de $\mathrm{T} 1$ recibieron forraje, de allí que las diferencias no estadísticamente significativas entre estos tratamientos permiten inferir que los abortos ocurrieron por causas no relacionadas con el $\beta$-caroteno.

El verano registró temperaturas mayores a $25{ }^{\circ} \mathrm{C}$, lo cual puede haber causado un efecto estresante en los cuyes. Quintela et al. (2008) indican que la principal función del $\beta$-caroteno en el organismo es su acción antioxidante, eliminando los radicales libres que tienen una mayor preferencia por el apa- rato reproductor, lo cual puede indicar un efecto beneficioso en épocas de extremo calor. Por otro lado, Aguiar-Zalzano y Rojas (2015) indican que los niveles de $\beta$-caroteno en el forraje varían según la época del año, siendo menores en forraje más secos; lo cual ocurre en el verano donde tienden a deshidratarse más rápido. Por tal motivo, la ocurrencia de abortos en este experimento podría estar relacionado al verano y la menor presencia de $\beta$-caroteno en el forraje.

Los resultados obtenidos para los parámetros tamaño de camada total, nacidas vivas y al destete siguieron la misma tendencia del Experimento I; sin embargo, en este experimento el tamaño de camada disminuyó considerablemente, pudiendo deberse al estrés por calor que provocó un incremento de la mortalidad embrionaria (Omtvedt et al., 1971; Ealy et al., 1993); asimismo, Chauca (1997) indica que los tamaños de camada en verano (2.90) son menores que en primavera (3.06). 
Cuadro 4. Efecto de la suplementación de $\beta$-caroteno en la dieta sobre el comportamiento productivo de cuyes de segundo parto

\begin{tabular}{|c|c|c|c|c|}
\hline \multirow{2}{*}{ Parámetros productivos } & \multicolumn{3}{|c|}{ Tratamientos } & \multirow{2}{*}{ P-value } \\
\hline & $\mathrm{T} 1$ & $\mathrm{~T} 2$ & T3 & \\
\hline \multicolumn{5}{|l|}{ Madres } \\
\hline $\begin{array}{l}\text { - Número de hembras } \\
\text { empadradas }\end{array}$ & 8 & 7 & 8 & \\
\hline $\begin{array}{l}\text { - Número de hembras que } \\
\text { parieron }\end{array}$ & 7 & 6 & 7 & \\
\hline - Peso al empadre (g) & $1352 \pm 85^{\mathrm{a}}$ & $1230 \pm 210^{\mathrm{a}}$ & $1288 \pm 62^{\mathrm{a}}$ & 0.59 \\
\hline - Peso al parto (g) & $1504 \pm 169$ & $1437 \pm 47$ & $1517 \pm 89^{\mathrm{a}}$ & 0.67 \\
\hline - Peso al destete (g) & $1580 \pm 109^{\mathrm{a}}$ & $1362 \pm 67^{\mathrm{b}}$ & $1557 \pm 57^{\mathrm{a}}$ & 0.04 \\
\hline - Mortalidad (\%) & $16.67 \pm 28.87^{\mathrm{a}}$ & $16.67 \pm 28.87^{\mathrm{a}}$ & $0^{\mathrm{a}}$ & 0.63 \\
\hline \multicolumn{5}{|l|}{ Crías } \\
\hline - Peso al nacimiento (g) & $117 \pm 15^{\mathrm{a}}$ & $114 \pm 10^{\mathrm{a}}$ & $125 \pm 13^{\mathrm{a}}$ & 0.54 \\
\hline - Peso al destete $(\mathrm{g})$ & $265 \pm 27^{\mathrm{a}}$ & $307 \pm 45^{\mathrm{a}}$ & $279 \pm 31^{\mathrm{a}}$ & 0.39 \\
\hline
\end{tabular}

${ }^{a, b}$ Letras diferentes dentro de filas indican diferencia estadística $(p<0.05)$

La mortalidad de las crías, tanto al nacimiento como al destete fueron mayores que las presentadas en el Experimento I. Se pudo observar mayores problemas durante el parto, posiblemente debido al exceso de calor, mientras que la mayor mortalidad al destete estaría relacionada al menor consumo de alimento de las madres, determinando un menor desarrollo de las crías.

No se encontraron diferencias significativas entre tratamientos en los parámetros productivos (Cuadro 4) a excepción del peso de madres al destete $(p<0.05)$, donde en T2 fue menor que en T1 y T3 que lograron mantener el peso al parto. Los cuyes de T2 presentaron una notoria pérdida de peso al destete, posiblemente relacionado al bajo consumo de alimento aunado al estrés por calor y al estrés de la gestación y del parto (Leal et al., 1996).

No hubo diferencias significativas entre tratamientos con respecto a la mortalidad de reproductoras, habiendo ocurrido en el último tercio de gestación y asociada a la presencia de abortos, que normalmente aumentan en la época de verano (Chauca et al., 2012).

Los pesos de las crías al nacimiento y al destete no presentaron diferencias significativas entre los tratamientos. Solórzano (2014) reportó pesos al nacimiento entre 166.5 y $172.5 \mathrm{~g}$ para sus sistemas de alimentación, sin diferencias significativas entre tratamientos. En forma similar, Alejandro (2016) reportó pesos de 166.3 y $157.5 \mathrm{~g}$ para crías de madres en su segundo parto sin diferencias significativas entre la alimentación mixta o integral. No obstante, sus resultados se encuentran por encima de lo observado en la presente investigación, posiblemente debido al menor consumo de alimento de las madres por el estrés calórico. El peso al destete fue ligeramente mayor en $\mathrm{T} 2$, pero esto se debe al menor tamaño de camada en este tratamiento. 


\section{ConClusiones}

La suplementación de $\beta$-caroteno a la dieta integral de cuyes reproductoras de primer parto en primavera o de segundo parto en verano, alimentadas con exclusión de forraje, no mejoró los parámetros reproductivos y productivos en comparación con dietas integrales solas o con forraje verde.

\section{Literatura Citada}

1. Aguiar-Zalzano E, Rojas A. 2015. Variaciones de â caroteno en sangre de vacas lecheras durante el periodo post parto. Nutr Anim Trop 9: 91-104. doi: 10.15517/nat.v9i2.21606

2. Alejandro P. 2016. Evaluación de niveles de energía digestible en dos sistemas de alimentación en reproducción de cuyes (Cavia porcellus). Tesis de Ingeniero Zootecnista. Lima, Perú: Univ. Nacional Agraria La Molina. $84 \mathrm{p}$.

3. Aréchiga CF, Ealy A D, Hansen P J. 1995. Evidence that glutathione in involved in thermotolerance of preimplantation murine embryos. Biol. Reprod 52: 1296-1301. doi: 10.1095/ biolreprod52.6.1296

4. Aréchiga CF, Staples CR, McDowell, LR, Hansen, PJ. 1998. Effects of timed insemination and supplemental $\beta$ carotene on reproduction and milk yield of dairy cows under heat stress. J Dairy Sci 81: 390-402. doi: 10.3168/jds.S00220302(98)75589-4

5. Badinga LR, Collier RJ, Thatcher $W W$, Wilcox CJ. 1985. Effect of climatic and management factor on conception rate of dairy cattle in subtropical environments. J Dairy Sci 68: 78-85. doi: 10.3168/jds.S0022-0302(85)80800-6

6. Besenfelder U, Solti L, Seregi J, Müller M, Brem G. 1995. Different roles for $\beta$-carotene and vitamin a in the reproduction on rabbits. Theriogenology 45: 1583-1591. doi: 10.1016/0093691X(96)00127-6
7. Chauca L. 1997. Producción de cuyes (Cavia porcellus). Estudio FAO: Producción y sanidad animal-138. [Internet]. Disponible en: http:// www.fao.org/ DOCREP/W6562s/W6562s00.htm

8. Chauca L. 2016. Similitudes y diferencias de los sistemas productivos en Perú y África, aportes desde el Perú. $1^{\circ} \mathrm{Se}-$ minario de Producción de cuyes en África: perspectivas y posibilidades de cooperación desde el Perú. Universidad Nacional Agraria La Molina, en La Molina, Lima.

9. Chauca L, Vergara R, Altamirano W, Reynaga M. 2012. Efecto de la alimentación en el crecimiento de cuyes sintéticos p- 0.6312 en verano e invierno en la costa central. INIA-UNALM. Revista Agro Enfoque N. ${ }^{\circ} 185$

10. Chew BP, Holpuch DM, O'Fallon. 1984. Vitamin $A$ and $\beta$-carotene in bovine and porcine plasma, liver corpora lutea, and follicular fluid. J Dairy Sci 67: 1316-1322. doi: 10.3168/jds.S00220302(84)-81439-3

11. Ealy A, Drost M, Hansen P. 1993. Developmental changes in embryonic resistance to adverse effects of maternal heats stress in cows. J Dairy Sci 76: 2899-2905. doi: 10.3168/jds.S00220302(93)77629-8

12. Graves-Hoagland RL, Hoagland T, Woody CO. 1988. Effect of $\beta$-carotene and vitamin $A$ on progesterone production by bovine luteal cells. J Dairy Sci 71: 1058-1062. doi: 10.3168/jds.S00220302(88)79652-6

13. Haliloglu S, Baspinar N, Serpek B, Erdem Haliloglu $S$, Baspinar $N$, Serpek B, Erdem H, Bulut Z. 1984. Vitamin a and $\beta$-carotene levels in plasma, corpus luteum and follicular fluid of cyclic and pregnant cattle. Reprod Domest Anim 37: 96-99. doi: 10.1046/ j.1439-0531.2002.00338.x

14. Lab Diet. 2014. Prolab guinea pig 5PI8. [Internet]. Available in: http:// www.labdiet.com/cs/groups/lolweb/ @1abdiet/documents/web_content/mdrf/ mdi4/ edisp/ducm04_028404.pdf 
15. Leal GP, Dorado LEV, España MMD. 1996. Efecto de la temperatura y humedad relativa en el comportamiento productivo del cuy (Cavia porcellus) en las fases de levante y engorde. Rev Cienc Agríc 14(1): 7-23.

16. Loven DP. 1988. A role for reduce oxygen species in heat induced cell killing and the induction of thermotolerance. Med Hypotheses 26: 39-50. doi: 10.1016/ 0306-9877(88)90111-9

17. Mamani T. 2015. Efecto de dos niveles de energía y dos sistemas de alimentación en cuyes (Cavia porcellus) reproductoras. Tesis de Ingeniero Zootecnista. Lima: Perú: Univ. Nacional Agraria La Molina. $129 \mathrm{p}$.

18. Matherne CM, Steffen EK, Wagner JE. 1987. Efficacy of commercial vaccines for protecting guinea pigs against Bordetella bronchiseptica pneumonia. Lab Anim Sci 37: 191-194.

19. Omtvedt IT, Nelson RE, Edwards RL, Stephens DF, Turman EJ. 1971. Influence of heat stress during early, mid and late pregnancy of gilts. J Anim Sci 32:312-317.

20. Pedraz F. 2001. Evaluación reproductiva de cuyes mejorados (Cavia porcellus) procedentes de Arequipa, Cajamarca y Lima. Tesis de Ingeniero Zootecnista. Lima, Perú: Univ. Nacional Agraria La Molina. 85 p.

21. Pickworth CL, Loerch SC, Kopec RE, Schwartz SJ, Fluharty FL. 2011. Concentration of pro-vitamin A carotenoids in common beef cattle feedstuffs. J Anim Sci 90: 1153-1561. doi: 10.2527/jas.2011-4217

22. Putney DJ, Mullins S, Thatcher WW, Drost M, Gross TS. 1989. Embryonic development in super ovulated dairy cattle exposed to elevated ambient temperatures between the onset of estrus and and insemination. Anim Reprod Sci 19: 37-51. doi: 10.1016/0378-4320(89)90045-6

23. Quintela L, Díaz C, Becerra J, Alonso G, Gracia S, Herradón P. 2008. Papel del $\beta$-caroteno y la vitamina A en la reproducción en el ganado vacuno: revisión. ITEA 3: 399-410.

24. Revilla J. 2011. Evaluación de la performance de cuyes suplementados con minerales orgánicos quelados en la fase de reproducción. Tesis de Ingeniero Zootecnista. Lima, Perú: Univ. Nacional Agraria La Molina. 98 p.

25. Schweigert FJ, Zucker H. 1988. Concentrations of vitamin A, $\beta$-carotene and vitamin $E$ in individual bovine follicles of different quality. J Reprod Fertil 82: 575-579.

26. Solórzano AJ. 2014a. Crianza, producción y comercialización de cuyes. Lima, Perú. Ed Macro. 93 p.

27. Solórzano AJ. 2014b. Evaluación de tres sistemas de alimentación comercial de cuyes (Cavia porcellus) en la etapa de reproducción. Tesis de Ingeniero Zootecnista. Lima: Univ. Nacional Agraria La Molina. $124 \mathrm{p}$.

28. Vergara RV. 2008. Avances en nutrición y alimentación en cuyes. En: XXXI Reunión Científica Anual de la Asociación Peruana de Producción Animal. Lima: APPA.

29. Wathes DC, Taylor VJ, Cheng Z, Mann GE. 2003. Follicle growth, corpus luteum function and their effects on embryo development in post-partum dairy cows. Reprod Suppl 61: 219-237. 